\title{
Les déterminants du choix des conteneurs plastiques dans la production des plants à Ouagadougou (Burkina Faso) : une contribution à la gestion des déchets plastiques
}

\author{
Yélézouomin Stéphane Corentin SOMÉ ${ }^{1,2 *}$, Eugénie KANGAMBÉGA ${ }^{2}$, Ali BÉNÉ ${ }^{3}$ et \\ Ignace DIENDÉRÉ ${ }^{3}$ \\ ${ }^{1}$ Unité de Formation et de Recherche en Lettres et Sciences Humaines (UFR-LSH), Département de \\ géographie, Université de Koudougou ; \\ ${ }^{2}$ Université Aube Nouvelle, \\ ${ }^{3}$ Centre National de Semences Forestières, Ministère de l'Environnement et des Ressources Halieutiques \\ *Auteur correspondant ; E-mail : some_y@yahoo.fr ; corentin.some@gmail.com
}

\section{RESUME}

Les déchets plastiques contribuent à la dégradation de l'environnement et affectent la santé humaine et animale. Les plastiques sont pourtant très utilisés comme conteneur par les pépiniéristes de la ville de Ouagadougou, pour la production de plants destinés au reboisement. Ce travail s'intéresse aux facteurs explicatifs du choix des types de conteneurs. Son objectif est de contribuer à une meilleure gestion des déchets de conteneurs plastiques issus de la production des plants par une meilleure connaissance des critères de choix des conteneurs des pépiniéristes de Ouagadougou au Burkina Faso. La méthodologie repose sur des enquêtes géographique et sociale et sur l'analyse des données (analyse de variance et test de corrélation). Ce travail a permis d'identifier deux groupes de conteneurs plastiques : celui provenant de matériaux de récupération et celui issu d'une production industrielle spécifique. Ces conteneurs plastiques sont des polyéthylènes (haute et basse densité) et des polypropylènes (simple et laminé). Les facteurs principaux de choix de conteneurs sont : le prix et la disponibilité. Les conteneurs les plus utilisés sont ceux qui sont les plus disponibles et ayant les coûts les plus bas. Ce travail pourrait être étendu à d'autres villes, aux moyens d'élimination des conteneurs usagés et à l'utilisation de conteneurs alternatifs.

(C) 2016 International Formulae Group. All rights reserved.

Mots clés : Pépiniériste ; conteneurs plastiques ; environnement ; Ouagadougou, Burkina Faso.

\section{The determiners of the choice of the plastic containers in the production of plants in Ouagadougou (Burkina Faso): a contribution to the management of plastic waste}

\begin{abstract}
Plastic waste contributes to the degradation of the environment and affects human and animal health. However, plastics are widely used as containers by Nursery men of the city of Ouagadougou, for the production plants intended for the reforestation. This work is interested in the explanatory factors of the choice of the types of containers. Its objective is to contribute to a better waste management of plastic containers stemming from the production of plants by a better knowledge of the criteria of choice of the containers of the
\end{abstract}


nursery gardeners of Ouagadougou to Burkina Faso. The methodology is based on geographic and social surveys and data analysis (analysis of variance and correlation test). This work allowed identifying two groups of plastic containers: that resulting from salvaged materials and that stemming from a specific industrial production. These plastic containers are polyethylene (high and low density) and polypropylenes (single and laminated). The main factors of choice of containers are: the price and the availability. The most used containers are the ones which are the most available and having the lowest costs. This work could be extended to other cities, to the ways of elimination of used containers and to the use of alternative containers.

(c) 2016 International Formulae Group. All rights reserved.

Keywords: Nurseryman; Plastic containers; the environment; Ouagadougou, Burkina Faso.

\section{INTRODUCTION}

La question des déchets plastiques, dans un contexte de préservation de l'environnement, est un véritable problème dans les pays en développement notamment en Afrique Subsaharienne. En effet, la mauvaise gestion de ces déchets (brûlage à l'air libre, dépôts non contrôlés...) entraîne des pollutions sur le sol, l'eau, l'air et nuit à la santé humaine et animale (Toguyeni, 2006 ; Doublier et al., 2009; Hien et al., 2010; Some et al., 2014). La production sans cesse croissante, corrélée avec la demande qui, ellemême est en rapport avec la croissance démographique, se révèle comme la principale difficulté pour mieux cadrer leur gestion (Kaboré, 2009 ; CNE, 2009 ; Lumami et al., 2016). L'utilisation des sachets plastiques est observée dans d'innombrables secteurs d'activités (alimentation, commerce, industrie, ...) entrainant sans doute une forte production de déchets (Adjagodo, 2016). Malgré l'existence de politiques et de stratégies bien définies en matière de gestion des déchets dans la plupart des pays en développement, leur application est source de controverse (Albiges, 2007; Amegnran, 2009). Au Burkina Faso, les ressources végétales connaissent de plus en plus une dégradation prononcée due à l'effet conjugué des activités anthropiques (défriche, exploitation abusive, feux, ...) et des facteurs climatiques notamment les sécheresses récurrentes (SP / CONEDD, 2010). Entre 1992 et 2000, cette déforestation était estimée à 360000 hectares par an (FAO, 2000). Cependant, les forêts constituent une source considérable de matériaux et de services divers contribuant significativement au bien-être et à la subsistance des sociétés humaines. Dans le souci de préserver et d'améliorer ce patrimoine forestier, des opérations de reforestation sont entreprises chaque année par plusieurs acteurs (gouvernement, ONG et particuliers), (Somé et al., 2016). Ces reboisements se font généralement par voie de plantation d'arbres entrainant une production à grande échelle des plants en pépinière. En effet, selon la Direction des Forêts (DIFOR), de 2005 à 2014 la production moyenne de plants était de 10720478 plants par an. Ce qui traduit l'utilisation d'un nombre approximativement équivalent de conteneurs plastiques.

À l'origine, les plants étaient produits à racines nues mais les résultats plus ou moins mitigés des projets de reboisement ont amené les services forestiers à envisager d'autres modes de production. Dans les zones sèches une plantation de pieds à racines nues a de moindre chance de reprise qu'une plantation de pieds en conteneurs. C'est ainsi que les plants sont désormais produits en protégeant les racines par des mottes soutenues par des conteneurs le plus souvent en sachets plastiques (Jeanicke, 2006). Après les plantations ou même pendant la production des plants (par exemple s'il y a rempotage), les conteneurs usés ne sont plus réutilisables et constituent ainsi une masse de déchets plastiques à évacuer. Cette étude s'inscrit dans la problématique de la préservation de la qualité de l'environnement et de la santé 
humaine et animale, dans le cadre des activités de production de plants. Elle se propose de réfléchir sur les facteurs explicatifs du choix des types de conteneurs en vue de la prévention des problèmes de pollutions issus d'une activité visant l'amélioration des écosystèmes.

L'objectif général de cette étude est donc de contribuer à une meilleure gestion des déchets de conteneurs plastiques par une meilleure connaissance des critères de choix des conteneurs par les acteurs. De façon spécifique, il s'agit (1) d'inventorier les types de conteneurs utilisés pour la production de plants à Ouagadougou, et (2) d'identifier les facteurs déterminant le choix de ces types de conteneur.

\section{MATERIEL ET METHODES}

\section{Présentation du site d'étude}

La ville de Ouagadougou située entre $12^{\circ} 14^{\prime}$ et $12^{\circ} 30^{\prime}$ de latitude Nord et $1^{\circ} 40^{\prime}$ et $1^{\circ} 24^{\prime}$ de longitude Ouest. Elle est limitée au nord par les communes rurales de Pabré et de Loumbila, à l'Est par celle de Saaba, au Sud par celle de Koubri et de Komsilga et enfin à l'Ouest par la commune rurale de Tanghin Dassouri. Elle couvre une superficie de 518 $\mathrm{Km}^{2}$, soit $0,2 \%$ du territoire national et est située approximativement au centre du pays, dans la province du Kadiogo (Figure 1). Elle est subdivisée en arrondissements et en secteurs. Le choix de la localité est effectué à la suite d'une étude exploratoire portant sur la quantité de plants produits chaque année dans le cadre des opérations de reboisement. Elle porte sur les campagnes de reforestation en prenant en compte et la gestion des déchets associée dans la zone d'étude.

\section{Matériel}

Le matériel utilisé est constitué d'un GPS Garmin 62S, des logiciels, GPS TrackMaker 13.9ArcGIS 10, Mini tab 15, Pack Office de Microsoft.

\section{Méthodes}

La méthodologie utilisée a été articulée autour de trois principaux points : une enquête géographie, une enquête sociale par questionnaire et l'analyse de données (analyse de variance et test de corrélation).

L'enquête géographique a permis de localiser les sites de production de plants de la ville de Ouagadougou à partir de coordonnées géographique pris à l'aide d'un GPS et de les caractériser. L'enquête sociale a été une enquête par questionnaire d'administration directe structurée autour des questions relatives à production des plants, aux producteurs et administrée aux producteurs de plants. Sur un total de 74 pépiniéristes repartis sur 8 sites inventoriés, 50 ont subi le questionnaire soit un taux d'échantillonnage de $67,56 \%$ (Tableau 1). La méthode d'échantillonnage est le tirage aléatoire sans remise. Les données obtenues après dépouillement du questionnaire ont analysées. Les méthodes d'analyses sont l'ANOVA (analyse de variance) et l'analyse de corrélation. Les principaux tests sont les tests de $\mathrm{Khi}^{2}\left(\mathrm{X}^{2}\right)$ et le coefficient de corrélation (r).

\section{RESULTATS}

\section{Les différents types de conteneurs plastiques}

L'inventaire des types de conteneurs a permis d'identifier deux grands groupes de conteneurs plastiques en fonction de la nature du matériau et de leur provenance. Chaque groupe comporte des types de conteneurs plastiques (Tableau 2).

Chaque groupe de conteneurs présente des particularités. C'est ainsi que les prix des conteneurs varient en fonction de la source d'approvisionnement, du type de matériau et de la taille (petits, moyens ou grands pots). La Figure 2 donne les coûts moyens des différents types de conteneurs plastiques recensés sur le terrain. Elle montre que les prix des conteneurs plastiques varient de 1 à 50 FCFA et que ce sont les conteneurs en sachets d'eau minérale qui sont les moins 
chers. Les plus chers sont les conteneurs en sac de ciment, suivi des pots plastiques spécifiquement fabriqués pour la production des plants.

Les enquêtes révèlent que hormis les conteneurs en sachet d'eau minérale, tous les autres types connaissent des temps de rupture. La majorité des pépiniéristes $(76 \%)$ estiment que les conteneurs en sachets d'eau minérale sont plus disponibles. Ces sachets d'eau minérale, du fait qu'ils sont beaucoup utilisés par la population, se trouvent un peu partout dans la ville ce qui explique le fait qu'ils peuvent être trouvés à n'importe quel moment. Les producteurs de plants évoquent en même temps leur facilité d'accès puissent qu'ils sont vendus par des femmes qui nettoient la ville ou des enfants qui les collectent et en revendent. Les plus difficilement disponibles sont les pots plastiques malgré qu'ils soient fabriqués à cette fin. Selon les pépiniéristes, ces pots sont produits par Faso Plast ou importés et ils sont constamment en manque au moment même où ils en ont le plus besoin.

\section{Les caractéristiques des pépiniéristes}

Les pépiniéristes présentent deux caractéristiques qui peuvent influencer leurs critères de choix des conteneurs. Il s'agit de leur niveau d'instruction (scolarisation) et de leur accès une formation sur le processus, sur les techniques et les outils de production de plants. Sur les cinquante (50) sujets enquêtés, plus de la moitié n'a pas été scolarisée ; moins de $30 \%$ ont un niveau scolaire et seulement quelques-uns ont atteint le niveau secondaire (14\%). Quant aux formations, les échanges ont révélé que la plupart des pépiniéristes (64\%) n'ont pas reçu de formation pour la production de plants. Ils ont appris le métier soit à travers les parents ou en imitant d'autres pépiniéristes. Les $36 \%$ autres ont reçu des formations en matière de production des plants.

\section{Les déterminants du choix des conteneurs}

Le choix d'un conteneur plastique dépend de plusieurs variables : sa capacité à s'adapter au développement du système racinaire de l'espèce à produire, sa facilité d'utilisation, ses dimensions, son accessibilité, sa disponibilité et son coût. Si tous ces critères sont évoqués pour une partie des enquêtés, deux parmi ces critères sont considérés par tous. Il s'agit de la disponibilité et du prix qui conditionnent l'accessibilité financière aux conteneurs et la rentabilité économique de l'activité.

La Figure 3 montre, d'une part que les conteneurs les plus utilisés par les pépiniéristes de la ville sont les moins chers. Ainsi, les matériaux de récupération d'industrie et du commerce dont les coûts compris entre 1 à 20 FCFA sont les plus utilisés. Il s'agit notamment des conteneurs en sachets d'eau minérale et les plastiques utilisés dans les mines avec des taux d'utilisation respectifs de $32,21 \%$ et $30,87 \%$. Les conteneurs en sacs de ciment, les pots plastiques spéciaux et les sachets de soude dont les prix moyens respectifs sont de 50, et 25 FCFA sont les moins utilisés. Le prix des conteneurs se présentent comme le facteur le plus déterminant de son utilisation. Le coefficient de corrélation entre le nombre de conteneurs utilisés et leur prix est 0,92. Ce qui est confirmé par le coefficient de corrélation calculé entre conteneurs plus utilisés et prix. Cette corrélation est forte et négative $(\mathrm{R}=$ 0,922199717) environ $92 \%$ de la variance du nombre d'utilisation d'un conteneur est corrélé au prix.

Il y a également un lien entre la disponibilité d'un conteneur et son utilisation. Le test du $\mathrm{Chi}^{2}$ (Pearson) effectué sur les conteneurs les plus disponibles et ceux les plus utilisé est statistiquement significatif $(7,0871 ; \mathrm{df}=2$ et $\mathrm{P}$ value de 0,0289$)$. On peut donc en déduire que les conteneurs les plus disponibles et les moins coûteux sont les plus utilisés. Cela motivé par la nécessité pour les pépiniéristes de faire face à la demande constante en plants et de faire profit. Pour cela il faut que les conteneurs soit constamment disponible et que leurs prix soit suffisamment bas. 


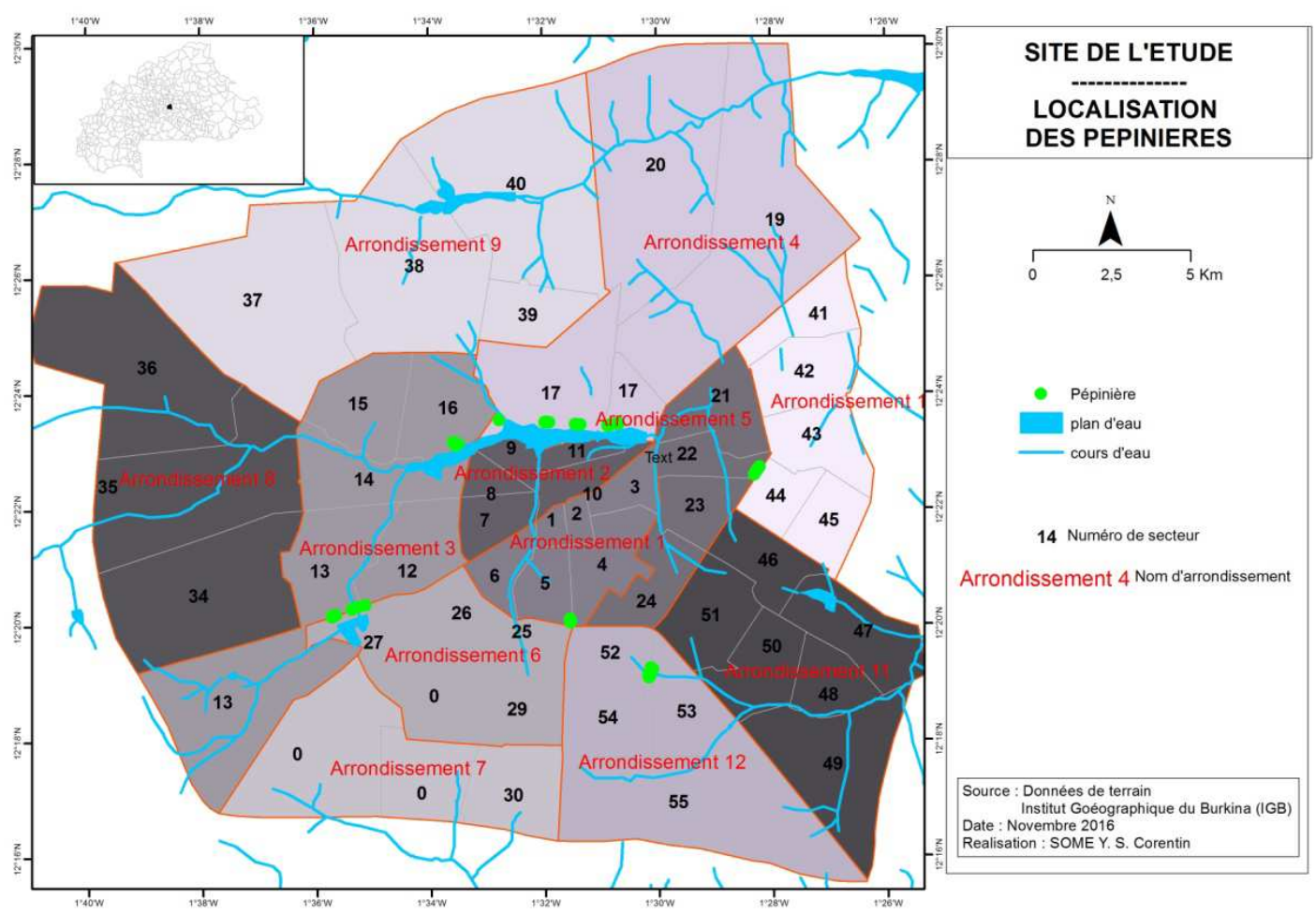

Figure 1 : Présentation du site.

Tableau 1: Répartition de l'échantillon.

\begin{tabular}{lc}
\hline Sites de localisation de pépinières & Nombre de pépinières choisies \\
\hline Barrage $\mathrm{n}^{\circ} 1$ & 5 \\
\hline Barrage $\mathrm{n}^{\circ} 2$ & 5 \\
\hline Barrage $\mathrm{n}^{\circ} 3$ & 12 \\
\hline Barrage de Boulmiougou & 6 \\
\hline Rond-point de la patte d'oie & 5 \\
\hline Monument des martyrs (Ouaga 2000) & 10 \\
\hline Mur du musée & 5 \\
\hline CNSF & 2 \\
\hline Total & $\mathbf{5 0}$ \\
\hline
\end{tabular}


Tableau 2 : Types de conteneurs utilisés pour la production de plants à Ouagadougou.

\begin{tabular}{lll}
\hline Groupes de conteneurs & Types de conteneurs & Caractéristiques \\
\hline $\begin{array}{l}\text { Conteneurs à usage essentiellement } \\
\text { sylvicole }\end{array}$ & Pots en plastique (sachets noirs) & $\begin{array}{l}\text { Polyétylène haute densité } \\
\text { (PEHD ou HDPE) }\end{array}$ \\
\hline & Sachet d'eau minérale & $\begin{array}{l}\text { Polyéthylène haute densité } \\
\text { et basse densité (PEHD et } \\
\text { PEBD) }\end{array}$ \\
\cline { 2 - 3 } \begin{tabular}{l} 
Matériaux de récupération d'industrie \begin{tabular}{l} 
et du commerce \\
\cline { 2 - 3 }
\end{tabular} \\
\cline { 2 - 3 }
\end{tabular} & Plastique utilisés dans les mines & $\begin{array}{l}\text { Polyéthylène basse densité } \\
\text { (PEBD ou LDPE) }\end{array}$ \\
\cline { 2 - 3 } & Emballage de soude & Polyéthylène (PE) \\
\cline { 2 - 3 } & Sac de ciment & Polypropylène (PP) laminé \\
\hline
\end{tabular}

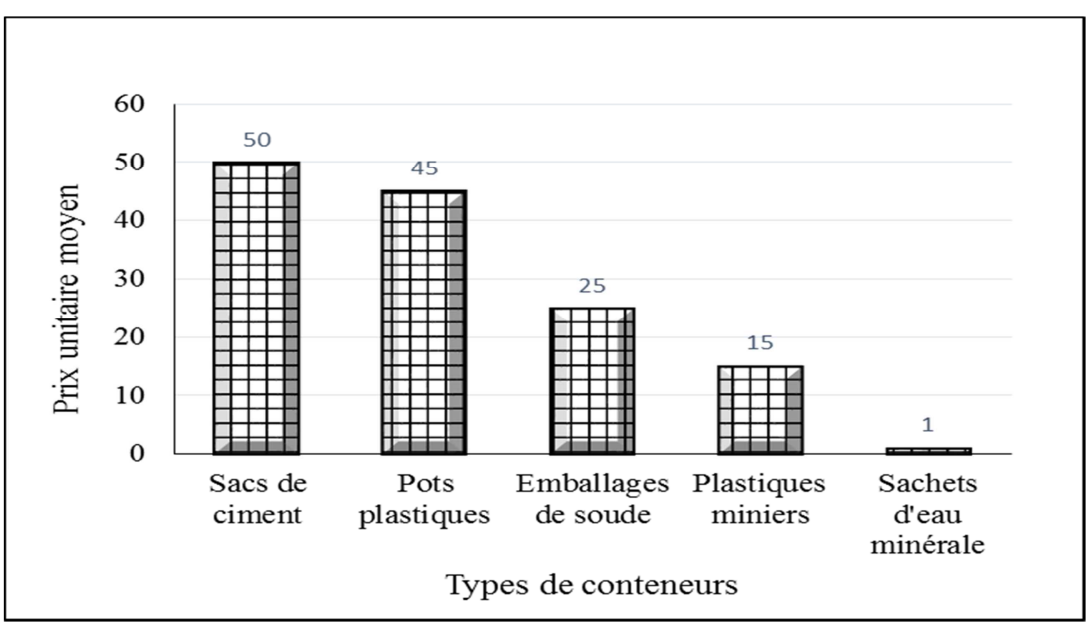

Figure 2: Types de conteneurs et leur prix moyen.

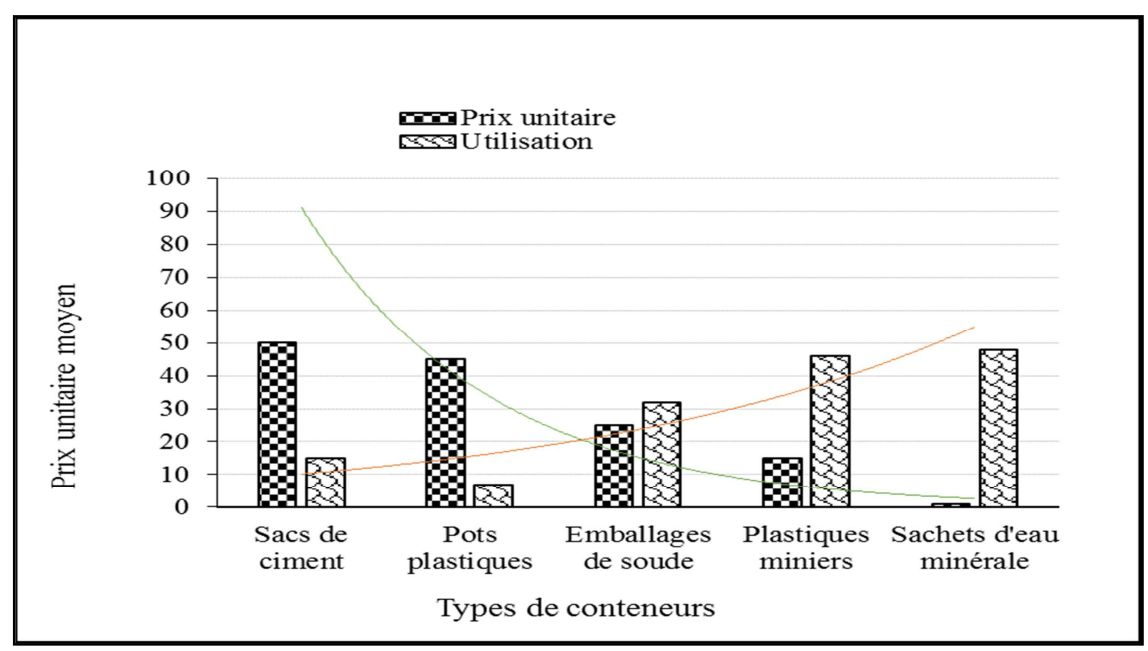

Figure 3 : Usage des conteneurs plastiques et leurs coûts. 


\section{DISCUSSION}

Plusieurs auteurs ayant travaillé sur des sujets similaires ont obtenu des résultats qui viennent renforcer les nôtres. En effet, d'après (Duryea, 1985; Le Bouler et al., 2011), le choix du type de conteneur est une étape importante dans le processus de production de plants de bonne qualité et dans la réussite des opérations de reboisement. Pour améliorer la survie et la croissance des plants, il faut des conteneurs adaptés. En phase avec nos travaux, Sanon (1987) et Jaenicke (2006) montrent que les conteneurs en sachets plastiques sont les plus communément utilisés dans les pépinières forestières dans les pays en développement et que l'utilisation d'un conteneur en pépinière est principalement conditionnée par sa disponibilité, son coût, sa performance. Tout compte fait, l'utilisation des types de conteneurs par les pépiniéristes est fortement liée à la disponibilité, l'accessibilité et surtout au prix. En dépit de l'existence des conteneurs spécialement fabriqués pour la production sylvicole (pots plastiques), les matériaux de récupération d'industrie et du commerce sont les plus utilisés. Bouju et Ouattara (2002) évoquent que ces matériaux de récupération sont produits en grande quantité dans les grands centres de consommation du pays, donc ils sont plus disponibles pour les citadins. Outre le prix, un conteneur, pour être d'une utilisation aisée doit satisfaire à des exigences d'ordre pratique et matériel. Certaines espèces en fonction de leurs caractéristiques intrinsèques de développement vont demander des conteneurs spécifiques, soit pour ses dimensions, soit pour sa durabilité (Sanon, 1987).

\section{Conclusion}

Pour la production de plants à Ouagadougou, les pépiniéristes utilisent des conteneurs généralement en sachets plastiques. La gestion de ces conteneurs plastiques est plutôt difficile compte tenu de leur nature. La présente étude, menée à Ouagadougou, a eu pour but d'approfondir la réflexion sur l'utilisation et l'élimination des conteneurs plastiques dans le processus de production de plants afin de contribuer à une meilleure gestion des déchets plastiques.

Cinq (05) types de conteneurs, tous sont en plastiques ont été inventoriés dans les pépinières de Ouagadougou. Ce sont, soit les pots plastiques fabriqués spécialement pour la production de plants, soit les matériaux de récupération d'industrie et du commerce.

Le coût et la disponibilité sont notamment les facteurs de choix qui motivent les pépiniéristes. Les conteneurs les moins et plus disponibles sont les plus utilisés.

$\mathrm{Ce}$ travail a mis en exergue les motivations dans pépiniéristes dans le choix du type de conteneur ainsi que la variété des conteneurs plastiques utilisés. Elle passe sous silence l'élimination de conteneurs usagée. Une étude approfondie de ces déchets en vue de leur élimination plus appropriée s'impose. La présente étude s'est limitée à Ouagadougou. Elle pourrait s'étendre à d'autres villes aux contextes écologiques et économiques différents.

\section{CONFLITS D'INTERETS}

Les auteurs déclarent ne pas avoir de conflits d'intérêts.

\section{CONTRIBUTION DES AUTEURS}

YSCS a assuré la conception du manuscrit, la rédaction de première version, la revue de littérature pour la discussion des résultats. EK a assuré la collecte et le traitement de données. $\mathrm{AB}$ a assuré le traitement des données statistiques et l'interprétation des résultats. ID a assuré l'organisation de la collecte de données sur le terrain. Tous les auteurs ont relu et corrigé le manuscrit.

\section{ACKNOWLEDGMENTS}

Les auteurs ont bénéficié de l'assistance et des conseils du Centre National des Semences Forestière du Burkina Faso qu'ils remercient vivement. Ils remercient également les pépiniéristes qui ont accepté de se soumettre à l'administration du 
questionnaire et qui ont fourni les informations nécessaires.

\section{REFERENCES}

Adjagodo A, Tchibozo MAD, Kelome NC, Lawani R. 2016. Flux des polluants liés aux activités anthropiques, risques sur les ressources en eau de surface et la chaine trophique à travers le monde: synthèse bibliographique. International Journal of Biological and Chemical Sciences, 10(3) : 1459-1472.

Albigès L. 2007. Gestion des déchets et assainissement à Fada N'Gourma (Burkina Faso). Deux réalités, un récit, Étude Récit n²0, Ouagadougou, Laboratoire Citoyennetés, 39p.

Amegnran YC. 2009. Problématiques de la gestion des déchets solides en Afrique. In Formation sur la gestion des déchets dans les villes africaines, 26 - 30 octobre 2009 Ouagadougou, CIFAL.

Bouju J, Ouattara F. 2002. Une anthropologie politique de la fange : conceptions culturelles, pratiques sociales et enjeux institutionnels de la propreté urbaine à Ouagadougou et Bobo-Dioulasso (Burkina Faso). SHADYCMarseille/GRIL-Ouagadougou.

CNE (Centre National D'Emballage, FRANCE), 2009. Emballages compostables et matériaux plastiques dits « Biodégradables » issus de ressources renouvelables. Note de position, Paris, 32 p.

Doublier G, Bowler-Ailloud M, Sorgho O. 2009. Valorisation des déchets de sachets plastiques. Utilisation comme liant dans la fabrication de matériaux composite : Application dans les villes subsahariennes. In Conférence Internationale sur la valorisation des déchets et de la Biomasse résiduelle dans les pays en développement, 9-11 Juillet 2009 - Ouagadougou, Burkina Faso.

Duryea ML. 1985. Evaluating seedling quality: principles, procedures and predictive ability of major tests. Res.
Lab., Oregon State University, Corvallis, OR; 143p.

FAO. 2000. Determination of the value of forests: Context, problems and orientations. Rome; 62.

Hien E, Favre-Bonte S, Masse D, Nazaret S. 2010. Impact de l'épandage de déchets urbains sur les communautés bactériennes de sols agricoles dans la périphérie de Ouagadougou, Burkina Faso. International Journal of Biological and Chemical Sciences, 4(5): 1721-1729 DOI:

http://dx.doi.org/10.4314/ijbcs.v4i5.6558 5

Jaenicke H. 2006. Bonnes pratiques de culture en pépinière forestière, Directives pratiques pour les pépinières de recherche. Manuel technique $\mathrm{n}^{\circ} 3$ - world agroforestry centre (ICRAF), $93 \mathrm{p}$.

Kabore SG. 2009. Les représentations sociales des déchets dans la ville de Ouagadougou : cas des déchets plastiques. Mémoire de maitrise en sociologie, Université de Ouagadougou, $100 \mathrm{p}$.

Kangambega E. 2015, gestion des dechets de conteneurs plastiques utilisés pour la production des plants : cas de la ville de ouagadougou, Mémoire de Master Management de l'Environnement et Développement Durable, Université Aube Nouvelle, 63 p.

Le Bouler H, Brahic P, Bouzoubaa Z, Achour A, Defaa C, Bellefontaine R. 2011. L'amélioration des itinéraires techniques en pépinière de production d'arganiers en mottes-conteneurs hors sol, Actes du Premier Congrès International de 1'Arganier, Agadir 15 - 17 Décembre, pp. 125-133.

Lumami K, Muyisa S, Jung CG. 2016. Contribution à l'état de lieux de déchets solides ménagers dans la ville D’Uvira, Sud-Kivu, République Démocratique du Congo. Int. J. Biol. Chem. Sci., 10(3): 1413-1421.

Sanou MD. 1987. Recherche sur quelques conteneurs utilisés en pépinière au Burkina Faso. Mémoire d'ingénieur des techniques du Développement rural / 
option : eaux et forêts, Institut du Développement Rural (IDR), 84 p.

Some YSC, Soro TD, Ouedraogo S. 2014. Étude de la prévalence des maladies liées à l'eau et influences des facteurs environnementaux dans l'arrondissement de Nomgr-Masson: cas du quartier Tanghin (Ouagadougou-Burkina Faso). International Journal of Biological and Chemical Sciences, 8(1): 289-303.

DOI

http://dx.doi.org/10.4314/ijbcs.v8i1.7

Somé YSC, Sondo R, Da DEC. 2016. Paradigm Change for a Better Vegetation Management in a Context of Land-cover
Deterioration: The Case of Gaoua District (Burkina Faso). Journal of Geography, Environment and Earth Science International. 5(2): 1-10. DOI: 10.9734/JGEESI/2016/23091

SP/CONEDD. 2010. Troisième Rapport sur l'État de l'Environnement au Burkina Faso, 247 p.

Toguyeni AR. 2006. La gestion des déchets solides de la ville de Ouagadougou (Burkina Faso) : État des lieux et analyse de la problématique des déchets d'emballages plastiques. Mémoire de fin d'études en gestion de l'environnement. Université Libre de Bruxelles, 130p. 